\title{
Cost/Charges Appraisal and Clinical Evidence Considerations in Orthopaedic Literature
}

\author{
Joe Sam Robinson $\mathrm{III}^{1}$, M. Sami Walid ${ }^{2}$, Waldo E. Floyd ${ }^{3}$, Joe Sam Robinson $\mathrm{Jr}^{4}$ \\ ${ }^{1}$ School of Medicine, Mercer University, Macon, USA; ${ }^{2}$ Kingsbrook Jewish Medical Center, Brooklyn, USA; ${ }^{3}$ OrthoGeorgia, Macon, \\ USA; ${ }^{4}$ Georgia Neurosurgical Institute, Macon, USA. \\ Email: mswalid@yahoo.com
}

Received March 20 ${ }^{\text {th }}$, 2012; revised April 28 ${ }^{\text {th }}$, 2012; accepted May $10^{\text {th }}, 2012$

\begin{abstract}
Introduction: Health care expenditures in the United States have rapidly risen in the last decade, including orthopaedic induced expenses. This paper addresses the methodology by which clinical evidence is obtained to better direct orthopaedic practice and encourage cost-efficiency. Questions: We conducted this inquiry to answer these questions: 1) Have orthopaedic expenses increased in the United States? 2) Does high grade clinical evidence prevail in orthopaedic literature? 3) Does clinical orthopaedic research include cost in outcome analysis? 4) Does the increase in orthopaedic expenses correlate with cost awareness in orthopaedic literature? Methods: The aggregate hospital charges (national bill) associated with three major orthopaedic procedures were extracted from the Nationwide Inpatient Sample (NIS) website (data available from 1997 to 2008). Using the biomedical search engine PubMed (launched 1996), different types of literature pertaining to general orthopaedic and three major orthopaedic procedures-hip replacement, knee replacement, and spine fusion, were probed regarding level of evidence and cost inclusion. Results: From 1997 to 2008, the national hospital charges for spine fusion increased by 10.4 times while for total knee replacement it increased by 4.9 times and for total/partial hip replacement by 3.4 times. From 1996 to 2010, PubMed indexed 1113 randomized controlled trials (RCTs) related to hip replacement, 942 related to knee replacement and 357 related to spine fusion. During the same period, RCTs related to total/partial hip replacement, total knee replacement and spine fusion procedures have increased by 3 times, 6 times, and 3.4 times, respectively. The percentage of blinded RCTS with cost analysis among all RCTs related to each procedure were $0 \%, 0.001 \%$ and $0.003 \%$ for total/partial hip replacement, total knee replacement and spine fusion procedures respectively. The correlation in the national hospital charges of all three procedures individually and their level I literature with cost element was not significant except for spine fusion. Conclusion: To improve literature shortfalls, substantial attention needs to be focused toward more rigorous studies which consider cost efficiency.
\end{abstract}

Keywords: Clinical Evidence; Randomized Clinical Trial; Blinded; Cost

\section{Introduction}

Health care expenditures in the United States have rapidly risen in the last three decades undergoing an eight fold increase from $\$ 253$ billion in 1980 to $\$ 2.3$ trillion in 2008 [1]. Although Americans benefit from these investments in healthcare, the recent economic slowdown and rising federal deficit are placing great strains on the systems used to finance health care. Unsustainable escalating healthcare costs therefore are threatening the viability of the American healthcare system [2]. Reflecting these general trends, orthopaedic surgery effectuated resource consumption has substantially increased [3].

While many factors including expert validation, local customs, malpractice concerns, insurance and government compensation opinions as well as practitioner experience and judgment influence clinical decisions, published clinical experience remains the standard reference point in clinical decision making. Evidence-based medicine is more and more replacing expert validation in the traditional sense as the sine qua non of generalized diagnostic and therapeutic authority.

It may be asserted that the highest level of evidence is required in order to practice evidence-based medicine [4]. This is particularly challenging in the field of surgical care, including orthopaedics. Randomized controlled trials provide level I evidence according to the United States Preventive Services Task Force definition. Sackett (2000) defined evidence-based management as the integration of best research evidence with clinical expertise and patient values [5]. In practice, healthcare practitioners must review the best research evidence, and inter- 
pret and compare the research with the current methods of practice [4].

To explore the methodology by which clinical evidence is obtained to better direct orthopaedic practice and also encourage cost-efficiency, we conducted the following inquiry trying to answer four main questions:

1) Have orthopaedic expenses increased in the United States?

2) Does high grade clinical evidence prevail in orthopaedic literature?

3) Does clinical orthopaedic research include cost in outcome analysis?

4) Does the increase in orthopaedic expenses correlate with cost awareness in orthopaedic literature?

\section{Search Strategies and Criteria}

Orthopaedic expenses were evaluated by querying the Nationwide Inpatient Sample (NIS) on the HCUPnet website. HCUPnet is an on-line query system that grants instant access to the largest set of publicly available allpayer health care databases. Using HCUPnet's easy stepby-step query system, tables and graphs on national and regional statistics and trends for community hospitals in the U.S. can be generated. The "national bill” (aggregate hospital charges) associated with three major orthopaedic procedures defined by their ICD-9 codes were extracted from the NIS data (available from 1997 to 2008):

- Total or partial hip replacement (ICD-9 code 81.51, 81.52).

- Total knee replacement (ICD-9 code 81.54).

- Spine Fusion (ICD-9 codes 81.00 - 81.08).

Orthopaedic literature was probed using a search engine. Several biomedical search engines exist in the World Wide Web; the most popular of them are the American PubMed and the European ScienceDirect. Using PubMed, different categories of literature pertaining to orthopaedic surgery starting from 1996 (date of PubMed launching) were explored using the criteria "English”, "Human” and the following keywords:

- "Orthopaedic" for orthopaedic literature.

- “Hip replacement”, "knee replacement”, “spine fusion” for three major orthopaedic procedures.

- "Blinded” for blinded studies indicating highest quality of scientific research.

- "Cost" as a marker of cost awareness in the literature.

- The Boolean operator "and” was used to narrow search and retrieve records containing all of the keywords it separates.

Special attention was paid to randomized controlled trials (RCTs) which constitute level I evidence in medical debates. It should be noted however that level II (cohort studies) and II evidence (consensus) has for long time been the only attained clinical evidence. For comparison purposes, we also investigated the amount of “orthopaedic" papers indexed as practice guidelines and case reports/series.

\section{Results}

In regard to orthopaedic expenses, the Nationwide Inpatient Sample (NIS) website shows that from 1997 to 2008, in synchrony with the rising national healthcare expenditures, hospital charges for spine fusion increased by 10.4 times while for total knee replacement it increased by 4.9 times and for total/partial hip replacement by 3.4 times (Figure 1). The summated hospital charges of all these procedures totaled $\$ 78.7$ billion in 2008. Increases in orthopaedic surgery effectuated hospital charges correlate with the national healthcare expenditures $(r=0.981, p$

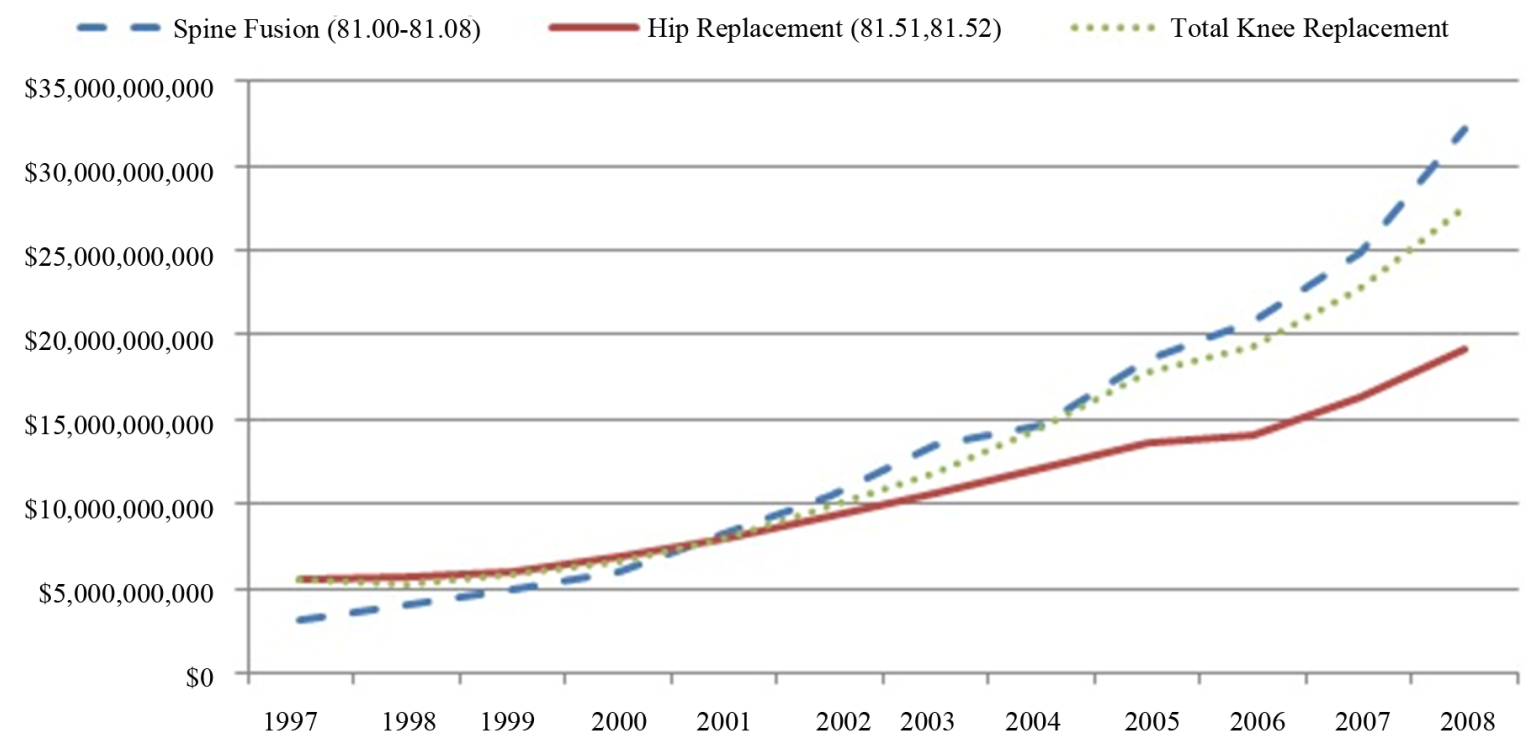

Figure 1. Shows the increase in total hospital charges (national bill) of three major orthopaedic procedures. 
$=0.000)$. To some degree such figures reflect increased orthopaedic implant use and expense.

Regarding the amount and quality of orthopaedic literature, during the period 1996-2010, PubMed indexed 73,948 orthopaedic papers, including 3041 (4.1\%) RCTs and 3094 clinical trials that did not fit the criteria for RCT. Of all orthopaedic RCTS, 63 (5.3\%) papers mentioned cost. The number of blinded orthopaedic RCTS with cost analysis among all orthopaedic RCTs was 11 (0.4\%, Figure 2). Practice guidelines papers related to orthopaedics increased during this period and totaled 77 papers; 4 (5.2\%) had a cost element. Case reports also increased and totaled 13745; $68(0.5 \%)$ mentioned cost. Of all orthopaedic literature from 1996 to 2010 (73,948), 2466 (3.3\%) involved cost. Analyzing orthopaedic level I literature, we see a parallel increase in the number of clinical trials, whether controlled randomized or other, from 1996 to 2010 (Figure 3). The increase in the number of blinded orthopaedic RCTS and RCTs with cost element from 1996 to 2010 was shallow.

Taking three major orthopaedic procedures individually, during the period 1996-2010, PubMed indexed 1113 RCTs related to hip replacement and 682 clinical trials that did not fit the criteria for RCT (Figure 4). For knee replacement, these numbers were 942 and 501 respectively and for spine fusion, 357 and 580 respectively
(Figures 5 and 6). Practice guidelines papers during this period totaled 15 for hip replacement, 7 for knee replacement, and 25 for fusion procedures. The percentage of blinded RCTS with cost analysis among all RCTs re-

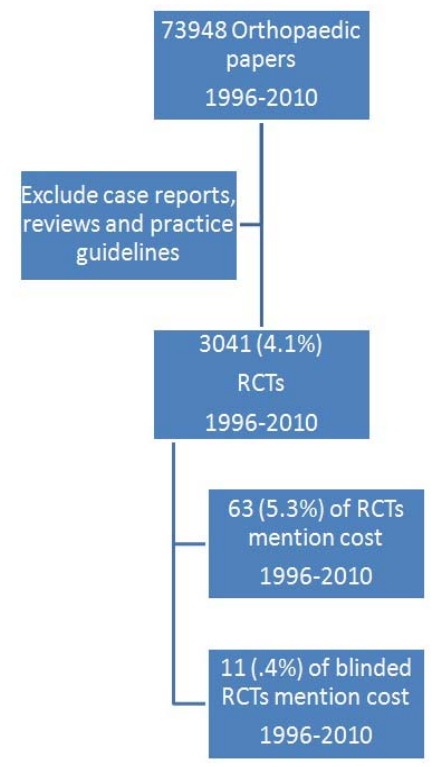

Figure 2. Shows a flow chart depicting the dearth of cost inclusion in high grade clinical osthopaedic evidence (RCTs).

Humans, English, Randomized Controlled Trial, English, Keyword: orthopedic.

-Humans, English, Randomized Controlled Trial, English, Keyword: orthopedic,blinded.

Humans, English, Randomized Controlled Trial, English, Keyword: orthopedic, cost.

-Humans, English, Clinical Trial except Randomized Controlled, English, Keyword: orthopedic.

-Humans, English, Randomized Controlled Trial, English, Keyword: orthopedic,blinded,cost.

Humans, English, Practice Guidelines, English, Keyword: orthopedic

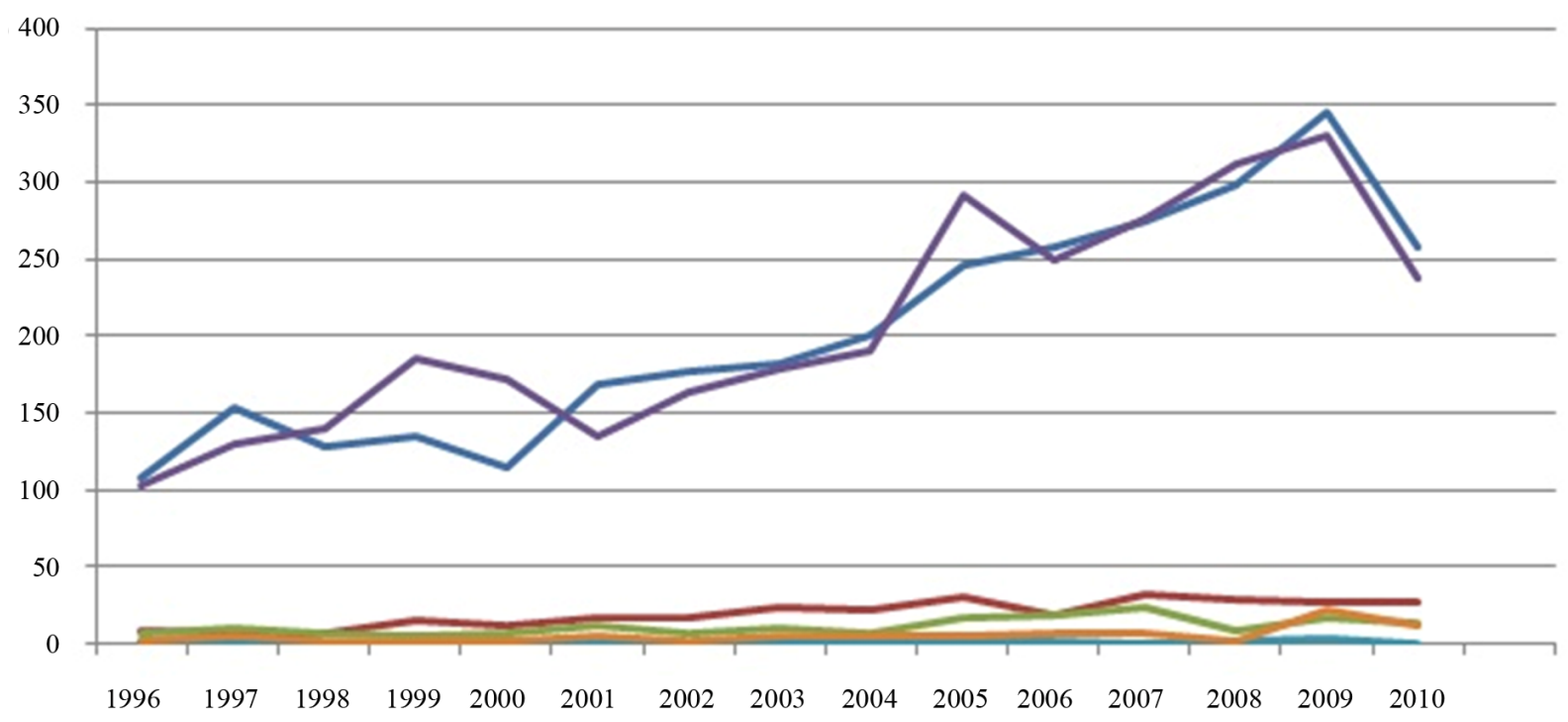

Figure 3 Shows the number of RCTS, Non-RCTS and practice guidelines related to orthopaedic surgery indexed per year. The dip at the end of the trend (2010) represents incomplete indexing by PubMed. 
- Humans, English, Randomized Controlled Trial, English, Keyword: hip replacement.

- Humans, English, Randomized Controlled Trial, English, Keyword: hip replacement,blinded.

- Humans, English, Randomized Controlled Trial, English, Keyword: hip replacement, cost.

- Humans, English, Clinical Trial except Randomized Controlled, English, Keyword: hip replacement.

Humans, English, Randomized Controlled Trial, English, Keyword: hip replacement,blinded,cost.

Humans, English, Practice Guidelines, English, Keyword: hip replacement.

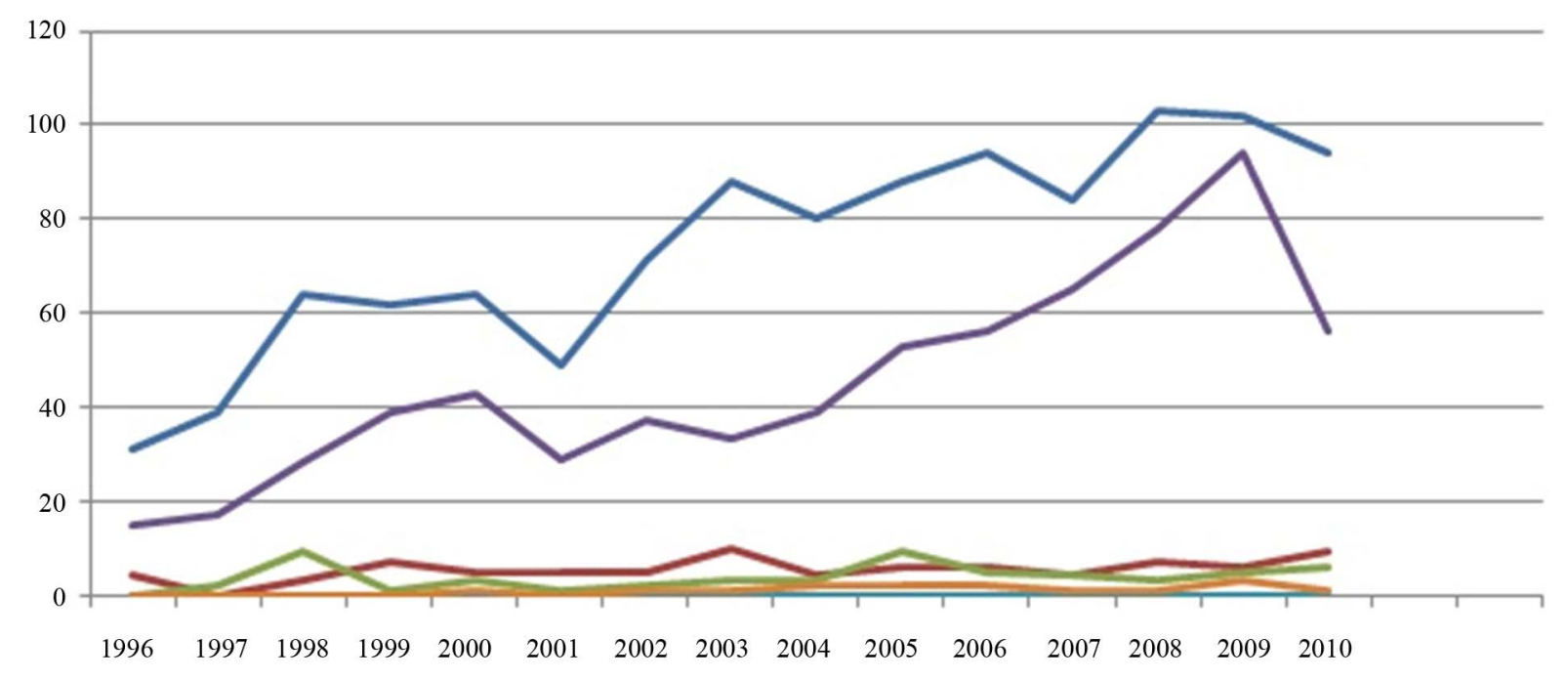

Figure 4. Shows the number of RCTS, Non-RCTS and practice guidelines related to hip replacement indexed per year. The dip at the end of the trend (2010) represents incomplete indexing by PubMed.

Humans, English, Randomized Controlled Trial, English, Keyword: knee replacement.

Humans, English, Randomized Controlled Trial, English, Keyword: knee replacement,blinded.

Humans, English, Randomized Controlled Trial, English, Keyword: knee replacement, cost.

Humans, English, Clinical Trial except Randomized Controlled, English, Keyword: knee replacement.

_ Humans, English, Randomized Controlled Trial, English, Keyword: knee replacement,blinded,cost.

Humans, English, Practice Guidelines, English, Keyword: knee replacement.

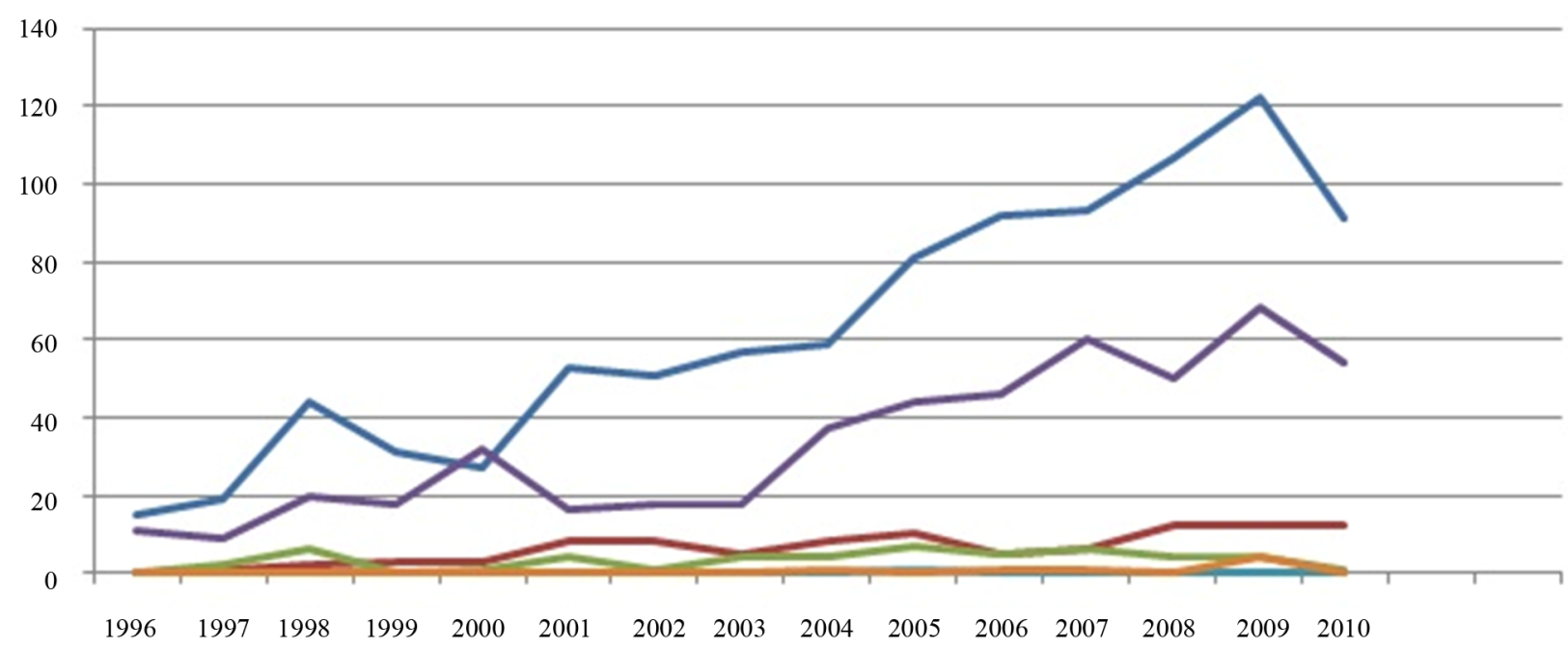

Figure 5. Shows the number of RCTS, Non-RCTS and practice guidelines related to knee replacement indexed per year. The dip at the end of the trend (2010) represents incomplete indexing by PubMed. 
lated to each procedure were $0 \%, 0.001 \%$ and $0.003 \%$ for total/partial hip replacement, total knee replacement and spine fusion procedures respectively. From 1996 to 2010, RCTs related to total/partial hip replacement, total knee replacement and spine fusion procedures have increased by 3 times, 6 times, and 3.4 times, respectively. However, the percentage of blinded RCTS and especially
RCTS with cost component did not significantly increase over the same period.

The correlation $(r=0.56, p=0.06$, Figure 7$)$ in the summated hospital charges of all three procedures together and their level I literature with cost analysis was not statistically significant. The correlation in the national hospital charges of all three procedures individually and

Humans, English, Randomized Controlled Trial, English, Keyword: spine fusion.

- Humans, English, Randomized Controlled Trial, English, Keyword: spine fusion, blinded.

- Humans, English, Randomized Controlled Trial, English, Keyword: spine fusion, cost.

Humans, English, Clinical Trial except Randomized Controlled, English, Keyword: spine fusion.

Humans, English, Randomized Controlled Trial, English, Keyword: spine fusion, blinded,cost.

Humans, English, Practice Guidelines, English, Keyword: spine fusion.

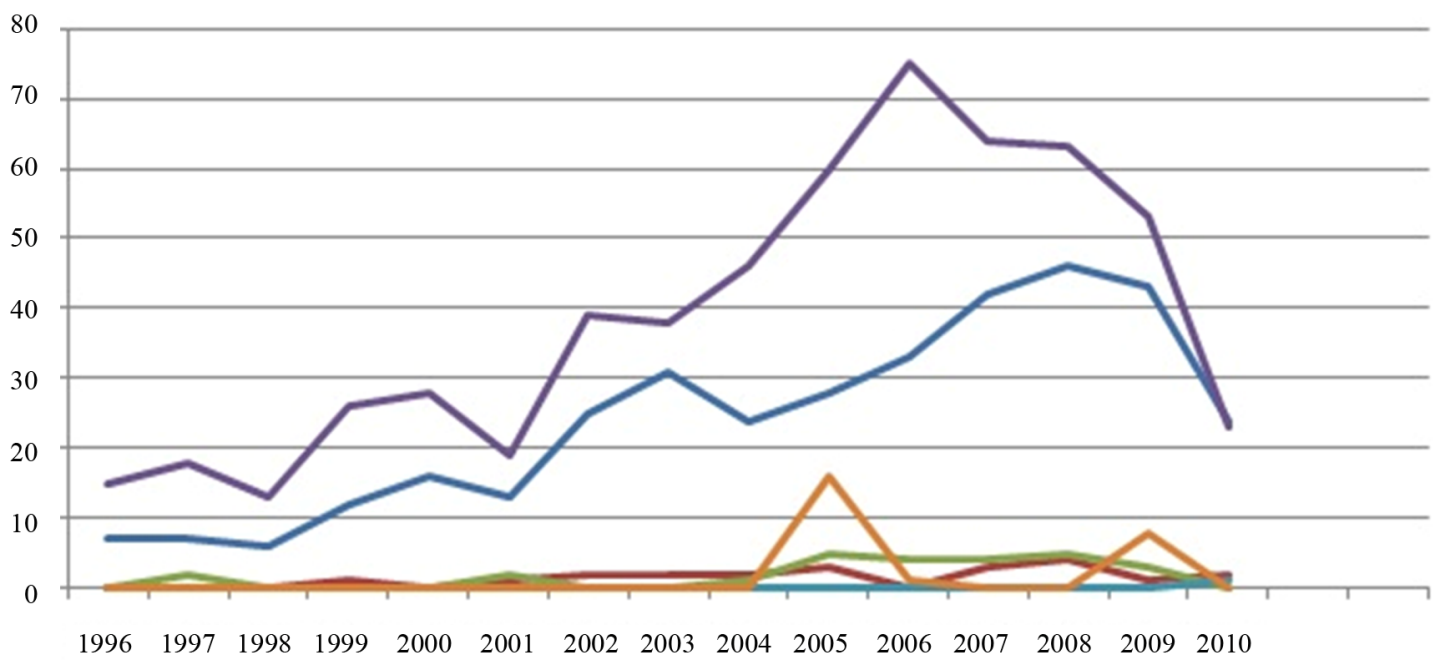

Figure 6. Shows the number of RCTS, Non-RCTS and practice guidelines related to spine fusion indexed per year. The dip at the end of the trend (2010) represents incomplete indexing by PubMed.
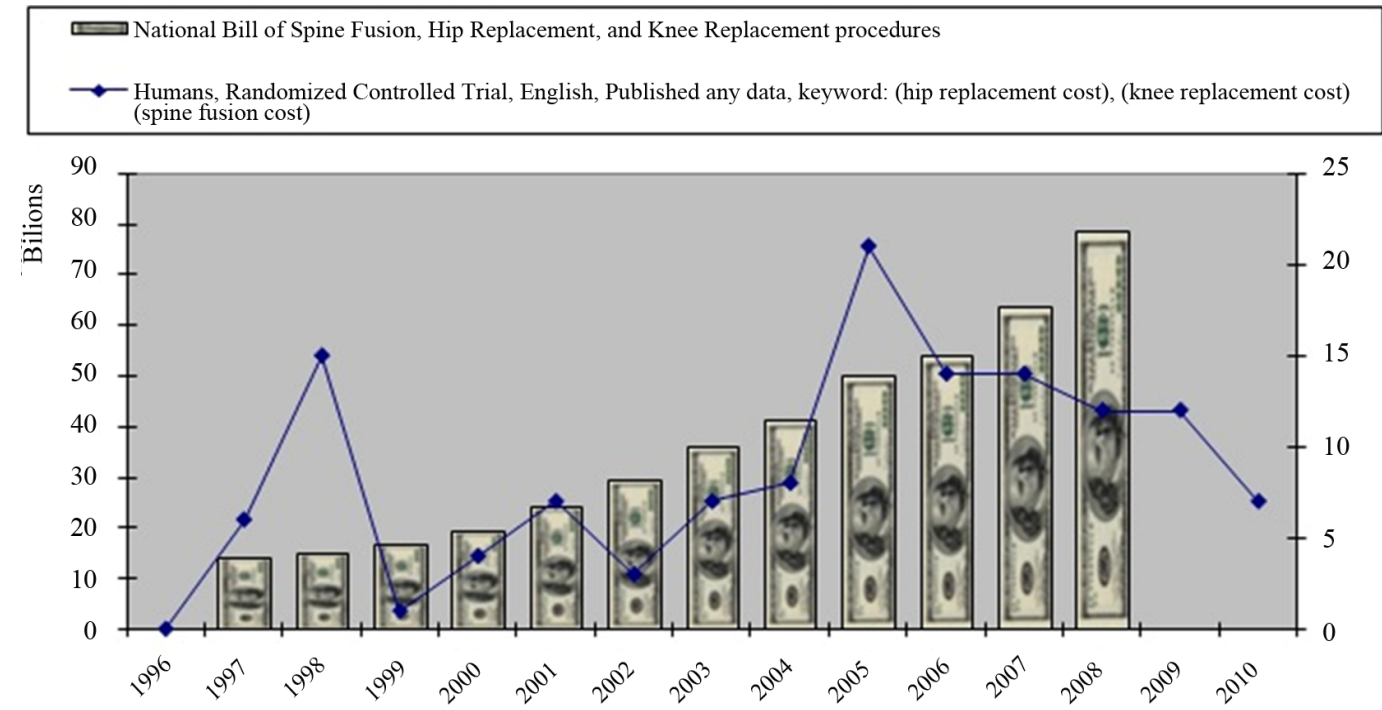

Figure 7. Shows the weak correlation between the rise in national bill and cost awareness in level I orthopaedic literature related to three major orthopaedic procedures $(r=0.56, p=0.06)$. 
their level I literature with cost element was not significant except for spine fusion $(r=0.791, p=0.002)$.

\section{Discussion}

Prompted by unsustainably soaring healthcare expenditures, we assessed orthopaedic expenses and pertinent literature with regard to level of evidence and cost awareness. However, our endeavors were hindered by several difficulties related to search engine querying technology. First, papers published in English language are not restricted to American sources. Second, keywords in PubMed do not always yield precise numbers of citations such as the use of the keyword "cost" does not necessarily stand for cost-analysis in retrieved papers. On the other hand, using summated national hospital charges associated with ICD-9 coded procedures as provided by the NIS is not a surrogate for hospital resource acquisition cost data-the summum bonum in any cost analysis. Moreover, gross hospital charges may be obscured by discounted prices negotiated by volume purchasers. Arguably lost wages, disability payments and legal expenses deserve also to be considered but are not easily accessible.

Though limited by such issues, hospital charges data disclose the crisis facing our national healthcare system [2,3,6-8]. The rise in orthopaedic induced hospital charges, while generally synchronizes with expanding national healthcare costs, in several circumstances were even greater. Interestingly, the rate of increase in summated national hospital charges for spine fusion over 11 years (10 fold from 1997 to 2008) as provided by the NIS website exceeded the increase rate in all national healthcare expenditures over 28 years (8 fold from 1980 to 2008). This aside, reasonably though not totally knowable, substantial benefits did arise from such expenditures. As the orthopaedic community is engendering such expenditures a discussion on cost-benefit analysis would seem to be particularly timely.

We found that only a small and static portion of orthopaedic literature is derived from high grade blinded clinical trials, arguably the basis for any sound clinical decision. Obviously, implementing such ambitious clinical studies is a difficult process requiring often the participation of multiple institutions (to obtain sufficient patient numbers) as well as considerable statistical and methodological guidance. Additionally, ethical constraints rendered such studies particularly inappropriate for many patient populations. Nor should one dismiss where circumstances dictate the appropriate use of less rigorous clinical trials.

One troubling observation was that cost data did not appear to receive proper attention in rigorously constructed clinical orthopaedic literature. Regrettably, the lack of such level I clinical evidence and cost consideration in the face of a national healthcare crisis encourages often hasty third party payor efforts at cost restraints [9] with the concomitant danger of capricious and arbitrary reduction in orthopaedic coverage. Correcting such shortfalls is not without difficulty as unfortunately even when requested cost data is not readily obtainable by interested physicians. The reliance placed upon the relatively few published high grade clinical studies would seem to mandate extra attention to such an important requisite.

Finally, absence of correlation between the curves of "cost" inclusion in high level orthopedic literature and summated hospital charges for three orthopedic procedures was observed. We believe that peer review journals should encourage improved clinical study methodology and cost analysis in processed manuscripts [10]. Additionally, separating cost-efficiency papers as an independent category of clinical research by biomedical search engines would ultimately activate physicians' involvement in superior healthcare efficiency despite increasing budgetary restraint. Periodic assessment of orthopaedic literature by use of available search engine technology might well redirect resources toward appropriate goals.

\section{REFERENCES}

[1] Centers for Medicare and Medicaid Services, Office of the Actuary, National Health Statistics Group, "National Health Care Expenditures Data,” January 2010.

[2] E. J. Emanuel and V. R. Fuchs, "The Perfect Storm of Overutilization," The Journal of the American Medical Association, Vol. 299, No. 23, 2008, pp. 2789-2791. doi:10.1001/jama.299.23.2789

[3] E. Stranges, C. A. Russo and B. Friedman, "Procedures with the Most Rapidly Increasing Hospital Costs, 2004-2007: Statistical Brief \#82,” In: Healthcare Cost and Utilization Project (HCUP) Statistical Briefs [Internet]. Agency for Health Care Policy and Research (US), Rockville, December 2006-2009.

[4] S. Ryan, L. Perrier and R. G. Sibbald, "Searching for Evidence-Based Medicine in Wound Care: An Introduction,” Ostomy Wound Management, Vol. 49, No. 11, 2003, pp. 67-75.

[5] D. L. Sackett, "The Fall of Clinical Research and the Rise of Clinical-Practice Research,” Clinical and Investigative Medicine, Vol. 23, No. 6, 2000, pp. 379-381.

[6] D. A. Sharan, J. Genuario, S. Mehta, S. Kusuma, A. Ranawat, R. Nunley and S. L. Weinstein, "Current Issues in Health Policy: A Primer for the Orthopaedic Surgeon,” Journal of the American Academy of Orthopaedic Surgery, Vol. 15, No. 2, 2007, pp. 76-86.

[7] H. A. Vallier, B. M. Patterson, C. J. Meehan and T. Lombardo, "Orthopaedic Traumatology: The Hospital Side of the Ledger, Defining the Financial Relationship between Physicians and Hospitals,” Journal of Orthopaedic Trauma, Vol. 22, No. 4, 2008, pp. 221-226. doi:10.1097/BOT.0b013e31815e92e5

[8] J. S. Cossman and D. Street, "Policy, Autonomy, and Physician Satisfaction," Journal of Health Care for the 
Poor and Underserved, Vol. 21, No. 3, 2010, pp. 898-912. doi:10.1353/hpu.0.0327

[9] R. Vesely, “Thinking Smaller in 2011. Insurers Expect Lower Profits as They Cope with Higher Costs, New Regulations,” Modern Healthcare, Vol. 41, No. 6, 2011, p. 14.
[10] D. J. Donegan, T. W. Kim and G. C. Lee, "Publication Rates of Presentations at an Annual Meeting of the American Academy of Orthopaedic Surgeons,” Clinical Orthopaedics and Related Research, Vol. 468, No. 5, 2010, pp. 1428-1435. doi:10.1007/s11999-009-1171-5 